\title{
RECURSIVE MULTIUSER DETECTION FOR DS-UWB SYSTEMS
}

\author{
Yihai Zhang, Wu-Sheng Lu, and T. Aaron Gulliver \\ Department of Electrical and Computer Engineering \\ University of Victoria \\ P.O. Box 3055 STN CSC \\ Victoria, B.C. V8W 3P6 CANADA \\ Email: $\{$ yhzhang, wslu, agullive $\} @$ ece.uvic.ca
}

\begin{abstract}
Multiple access UWB communications has recently attracted a great deal of attention. In a multiple access UWB system, multiuser detection is desirable to effectively reduce multiple access interference (MAI). A suboptimal ML detector based on a recursive convex relaxation strategy is proposed for DS-UWB systems in this paper. This algorithm employs a recursive approach to deduce a sequence of reduced-size quadratic problems, in each of which only the most probable information bits are detected. Performance results are presented which show that the proposed detector provides near-optimal performance relative to the ML detector with considerably reduced computational complexity.
\end{abstract}

\section{INTRODUCTION}

The Federal Communications Commission (FCC) defines ultra wideband (UWB) technology as a wireless transmission system that occupies a fractional bandwidth $B_{f} \geq 20 \%$, where $B_{f}=2 \frac{f_{H}-f_{L}}{f_{H}+f_{L}}, f_{H}$ and $f_{L}$ are the higher and lower $-10 \mathrm{~dB}$ frequencies, or more than $500 \mathrm{MHz}$ of absolute bandwidth [1]. UWB technology has the ability to provide high data rates at low cost and with low power consumption. It utilizes very short pulses (on the order of a nanosecond or less) in transmission, thus it is also known as impulse radio transmission. Pulse amplitude modulation (PAM) or pulse position modulation (PPM) are commonly used in UWB systems. Due to the extremely short duration of UWB pulses, UWB systems possess several desirable properties such as multipath immunity, interference suppression, and low complexity detection.

Multiple access UWB communications has recently received a great deal of attention in the research community. This includes time hopping UWB (TH-UWB) [2] and directsequence UWB (DS-UWB) [3] technologies. As with DSCDMA, users are separated by independent pseudorandom (PR) sequences in a DS-UWB system. In principle, a matched filter and a rake receiver are sufficient to suppress channel impairments. However, in a multiple access system, multiuser detection is desirable to effectively reduce or eliminate multiple access interference (MAI). Similar to DS-CDMA systems, maximum-likelihood (ML) and minimum mean square error (MMSE) detectors can be developed for DSUWB systems [4].

In this paper, we propose a suboptimal ML detector based on a recursive convex relaxation strategy for DS-UWB systems. First, the combinatorial problem associated with the ML detector is relaxed into a convex quadratic minimization problem. A recursive approach is then employed to deduce a sequence of reduced-size quadratic problems, in each of which only the most probable information bits are detected. Performance results are presented which illustrate that the proposed detector provides near-optimal performance relative to the ML detector with significantly reduced computational complexity.

This paper is organized as follows. Section 2 describes the DS-UWB system model. The recursive convex quadratic programming (QP) minimization algorithm is presented in Section 3. Performance results are given in Section 4, and some conclusions are given in Section 5.

\section{SYSTEM MODEL}

We consider a $K$-user DS-UWB system transmitting over an AWGN channel. Each user employs binary PAM (BPAM) direct sequence spread spectrum modulation. For simplicity, we only consider synchronous DS-UWB systems.

In a DS-UWB system, each information bit is spread over multiple pulses to achieve processing gain. Thus, the UWB pulses act like the PR sequence waveform in a DSCDMA system. The pulse waveform used in this paper is Scholtz's monocycle, which approximates to the second derivative of the Gaussian pulse and is given by [5]

$$
p(t)=A\left[1-4 \pi\left(\frac{t-t_{d}}{\tau_{m}}\right)^{2}\right] \exp \left[-2 \pi\left(\frac{t-t_{d}}{\tau_{m}}\right)^{2}\right]
$$


where $t_{d}$ denotes the center of the pulse and $\tau_{m}$ is the pulse shape parameter.

With $p(t)$ defined in (1), the transmitted waveform can be expressed as

$$
s_{k}(t)=\sum_{j=1}^{N} c_{k}(j) p\left[t-(j-1) T_{c}\right], \quad \text { for } t \in\left[0, T_{b}\right]
$$

where $T_{b}$ is the symbol duration, $T_{c}$ is the chip duration, and $\mathbf{c}_{\mathbf{k}}=\left[c_{k}(1) c_{k}(2) \cdots c_{k}(N)\right]^{T}$ with $c_{k}(i) \in\{-1,1\}$ is the spreading sequence assigned to the $k$ th user. For DSUWB PAM systems, $T_{b}=N T_{c}=N T_{p}$ where $T_{p}$ denotes the pulse duration. With BPAM modulation, the information bits $b_{k}$ are binary antipodal signals, each of which assumes value -1 or 1 with equal probability. We also assume that the transmitted waveforms are normalized, i.e.,

$$
\left\|s_{k}(t)\right\|^{2}=\int_{0}^{T_{b}} s_{k}^{2}(t) d t=1 \text { for } k=1,2, \ldots, K
$$

The received signal is a combination of the transmitted signals from all users plus channel noise, and can be expressed as

$$
r(t)=\sum_{i=0}^{\infty} \sum_{k=1}^{K} A_{k}(i) b_{k}(i) s_{k}\left(t-i T_{b}\right)+n(t)
$$

where $A_{k}$ is the signal amplitude of the $k$ th user, and $n(t)$ is white Gaussian noise with zero mean and variance $\sigma^{2}$.

\section{RECURSIVE MULTIUSER DETECTOR}

It is well known that the output of a matched filter possesses sufficient statistics to make an optimal decision [6]. For an AWGN channel with MAI, the output of the matched filter can be expressed in vector form as

$$
\mathbf{y}=\mathbf{R A b}+\tilde{\mathbf{n}}
$$

where $\mathbf{R}=\mathbf{S}^{T} \mathbf{S}=\left\{\rho_{j k}\right\}$ is the normalized cross-correlation matrix with $\mathbf{S}=\left[\begin{array}{llll}\mathbf{s}_{1} & \mathbf{s}_{2} & \cdots & \mathbf{s}_{K}\end{array}\right]$ where $\mathbf{s}_{k}$ is the signature waveform of $k$ th user, and $\rho_{j k}=\int_{0}^{T_{b}} s_{j}(t) s_{k}(t) d t$. $\mathbf{A}=\operatorname{diag}\left\{A_{1}, A_{2}, \ldots, A_{K}\right\}, \mathbf{b}=\left[\begin{array}{llll}b_{1} & b_{2} & \cdots & b_{K}\end{array}\right]^{T}$, and $\tilde{\mathbf{n}}=\left[\begin{array}{llll}\tilde{n}_{1} & \tilde{n}_{2} & \cdots & \tilde{n}_{K}\end{array}\right]^{T}$ denotes a zero mean Gaussian random process with covariance $E\left[\tilde{\mathbf{n}} \tilde{\mathbf{n}}^{T}\right]=\sigma^{2} \mathbf{R}$.

The ML detector maximizes the joint posteriori probability by determining the information bearing waveform that is closest to the observed waveform in terms of Euclidean distance [6]. The ML detector can be obtained by solving the optimization problem

$$
\begin{aligned}
& \operatorname{minimize} \mathbf{x}^{\mathbf{T}} \mathbf{H} \mathbf{x}+\mathbf{x}^{\mathbf{T}} \mathbf{p} \\
& \text { subject to }: x_{i} \in\{-1,1\} \text { for } i=1,2, \ldots, K
\end{aligned}
$$

where $\mathbf{H}=\mathbf{A R A}, \mathbf{p}=-\mathbf{2} \mathbf{A y}$, and $x_{i}$ is the $i$ th component of $\mathbf{x}$.

\subsection{Recursive ML Detection Algorithm}

The amount of computation required to solve the problem in (6) increases exponentially with the number of users involved, and becomes prohibitive even for moderate $K$. In this paper, we seek to find a suboptimal solution of (6) by considering the following relaxed constrained optimization problem

$$
\begin{aligned}
& \operatorname{minimize} \mathbf{x}^{\mathbf{T}} \mathbf{H} \mathbf{x}+\mathbf{x}^{\mathbf{T}} \mathbf{p} \\
& \text { subject to : }-1 \leq x_{i} \leq 1 \text { for } i=1,2, \ldots, K .
\end{aligned}
$$

A desirable feature of problem (7) is that it is a convex quadratic programming $(\mathrm{QP})$ problem whose global solution can be found with polynomial-time computational complexity [7]. A similar approach was attempted recently for multiuser detection for CDMA systems [8]. The main purpose of this paper is to demonstrate that convex relaxation can also be applied to DS-UWB systems.

Our multiuser detection algorithm is a recursive algorithm on the basis of convex QP formulation (7). The index set of detected information bits before the $j$ th iteration is defined as $\Omega_{j}$, where $\bar{b}_{i}, i \in \Omega_{j}$ is the $i$ th detected information bit. In the $j$ th iteration, we have a convex QP problem of the form (7), i.e.,

$$
\begin{aligned}
& \operatorname{minimize} \mathbf{w}^{T} \mathbf{H}_{j} \mathbf{w}+\mathbf{w}^{T} \mathbf{q}_{j} \\
& \text { subject to : }-1 \leq w_{i} \leq 1 \text { for } i=1,2, \ldots, K_{j}
\end{aligned}
$$

where $\mathbf{H}_{j}$ and $\mathbf{q}_{j}$ are obtained from the original data, $\mathbf{H}$ and $\mathbf{p}$, respectively, but with reduced size. On solving the convex QP problem in (8), the magnitudes of the components of the solution $\mathbf{w}$ are examined. If $\left|w_{i}\right|$ exceeds a given threshold $\alpha$, the corresponding information bit is declared to be detected as $\operatorname{sign}\left(w_{i}\right)$; otherwise component $w_{i}$ remains undetermined and will be considered as a design variable in the next iteration. Based on this examination, a QP problem similar to that in (8) of reduced size is formulated where the variable vector $\mathbf{w}$ contains all undetected information bits. The iterations continue until all information bits have been identified.

The relationship between $\mathbf{H}_{j}, \mathbf{q}_{j}$ in (8) and $\mathbf{H}, \mathbf{p}$ in (7) has been discussed in [8], and is given by

$$
\mathbf{x}^{T} \mathbf{H} \mathbf{x}+\mathbf{x}^{T} \mathbf{p}=\mathbf{w}^{T} \mathbf{H}_{j} \mathbf{w}+\mathbf{w}^{T}\left(\mathbf{p}_{j}+2 \tilde{\mathbf{H}}_{j} \overline{\mathbf{b}}_{j}\right)+\text { const }
$$

where $\mathbf{H}_{j}$ denotes the submatrices of $\mathbf{H}$ obtained by removing the rows and columns of $\mathbf{H}$ whose indices are in $\Omega_{j}$, and $\tilde{\mathbf{H}}_{j}$ is obtained by removing the rows of $\mathbf{H}$ whose indices are in $\Omega_{j}$ and the columns of $\mathbf{H}$ whose indices are not in $\Omega_{j} ; \mathbf{p}_{j}$ denotes the vectors obtained by removing the components of $\mathbf{p}$ whose indices are in $\Omega_{j}$; const is a constant 
that depends on quantities and not related to w. From (5), (6) and (8), $\mathbf{H}_{j}, \tilde{\mathbf{H}}_{j}, \mathbf{p}_{j}$ and $\mathbf{q}_{j}$ can be expressed as

$$
\begin{aligned}
\mathbf{H}_{j} & =\mathbf{A}_{j} \mathbf{S}_{j}^{T} \mathbf{S}_{j} \mathbf{A}_{j} \\
\tilde{\mathbf{H}}_{j} & =\mathbf{A}_{j} \mathbf{S}_{j}^{T} \overline{\mathbf{S}}_{j} \overline{\mathbf{A}}_{j} \\
\mathbf{p}_{j} & =-2 \mathbf{A}_{j} \mathbf{S}_{j}^{T} \mathbf{r} \\
\mathbf{q}_{j} & =\mathbf{p}_{j}+2 \tilde{\mathbf{H}}_{j} \overline{\mathbf{b}}_{j}
\end{aligned}
$$

where $\mathbf{S}_{j}$ and $\overline{\mathbf{S}}_{j}$ denote the submatrices of $\mathbf{S}$ that remove the columns of $\mathbf{S}$ whose indices are in $\Omega_{j}$ and not in $\Omega_{j}$, respectively, [8].

A step-by-step description of the proposed iterative multiuser detection algorithm is given below, where $\mathbf{w}^{*}$ denotes the solution vector of the convex QP problem (8), and $w_{i}^{*}$ is the $i$ th component of $\mathbf{w}^{*}$.

Parameters: $n_{j} \geq j-1$ is the number of information bits detected before the $j$ th iteration. $\Omega_{j}$ is the index set of the bits detected before $j$ th iteration. $\alpha$ is a given threshold.

Initialization: $j=1, n_{1}=0, \Omega_{1}=$ null, $\mathbf{H}_{1}=\mathbf{H}$, $\mathbf{q}_{1}=p, \overline{\mathbf{b}}_{1}=0$, and $\tilde{\mathbf{H}}_{1}=0$.

Step 1: Solve the QP problem

$$
\begin{aligned}
\operatorname{minimize} & \mathbf{w}^{T} \mathbf{H}_{j} \mathbf{w}+\mathbf{w}^{T} \mathbf{q}_{j} \\
\text { subject to: } & -1 \leq w_{i} \leq 1 \text { for } i \notin \Omega_{j}
\end{aligned}
$$

denotes the solution as $\mathbf{w}^{*}$.

Step 2: If $\left|w_{i}^{*}\right| \geq \alpha$, let $\operatorname{sign}\left(w_{i}^{*}\right)$ be the binary information bit corresponding to $w_{i}^{*}$.

Step 3: Update $\Omega_{j}$ to $\Omega_{j+1}$, which includes all information bits detected previously. Update $n_{j+1}$, if $n_{j+1}=K$, stop and output $\overline{\mathbf{b}}_{j}$ as the detected information vector; otherwise go to Step 4.

Step 4: Update $\overline{\mathbf{b}}_{j+1}, \mathbf{H}_{j+1}, \tilde{\mathbf{H}}_{j+1}, \mathbf{p}_{j+1}$ and $\mathbf{q}_{j+1}$ according to (15). Set $j=j+1$ and repeat from Step 1.

Note that in Step 2, if there are no information bits that exceed the threshold, the $w_{i}^{*}$ with maximum magnitude is chosen, and $\operatorname{sign}\left(w_{i}^{*}\right)$ is claimed as the binary information bit corresponding to $w_{i}^{*}$. In this way, we ensure that at least one information bit is detected each iteration, and all information bits will be detected in at most $K$ iterations. The threshold may affect the performance of the recursive convex programming (RCP) multiuser detector. A small threshold may degrade the performance of the RCP detector, but the number of iterations is also reduced. Conversely, a larger threshold may improve performance, but the number of iterations is increased.

\section{NUMERICAL RESULTS}

Simulations were carried out to evaluate the performance of the proposed recursive convex programming multiuser detector (RCP-MUD). Based on the bit error rate (BER) and computational complexity, we compare the results with conventional ML, MMSE and convex programming (CPMUD) detectors. For the CP-MUD detector, decisions are made by solving the problem (7) and the sign of the solution is the decision on the information bits.

The RCP-MUD detector was implemented using the MATLAB routine quadprog. Since BPAM is used, the multiuser detector must determine the information bits transmitted. A relatively large convergence tolerance $10^{-2}$ was chosen for quadprog to keep the number of iterations low. Gold sequences were used for the spreading sequences, UWB pulse duration was set to $0.5 \mathrm{~ns}$. All users were assumed to have equal power, and $10^{5}$ simulation runs were performed to evaluate the average BER performance. The threshold $\alpha$ used in the RCP-MUD detector was 0.8 .

Fig. 1 presents the average BER performance with eight users and matched filter, ML, MMSE, CP-MUD and RCPMUD detectors. As the number of users is sufficiently large, the MAI cannot be eliminated by the matched filter efficiently, thus a multiuser detector is desirable. It can be observed that the RCP-MUD detector outperforms the MMSE and CP-MUD detectors, and offers near-optimal performance close to that of a ML detector.

The computational complexity of the ML, CP-MUD and RCP-MUD detectors in terms of CPU time on a SUN Blade150 workstation is plotted in Fig. 2. This shows that as the number of users increases, the time required for the RCP-MUD detector increases with polynomial order, whereas that for the ML detector increases exponentially as $O\left(2^{K}\right)$. The computational complexity of the RCP-MUD is much lower than that of ML detector when the number of users is large. Furthermore, the RCP-MUD detector requires more computation than that of the CP-MUD detector as the RCP-MUD detector typically needs more iterations to detect the information bits.

\section{CONCLUSIONS}

In this paper, a suboptimal ML detector for DS-UWB systems was presented based on a recursive convex relaxation algorithm. The combinatorial problem associated with the ML detector was relaxed into a convex quadratic minimization problem. Then a recursive approach was employed to solve a sequence of reduced-size quadratic problems and approximate the ML detection solution. Our performance results show that the proposed algorithm outperforms both the MMSE and CP-MUD detectors. In addition, it is able to offer near-optimal performance close to that of the ML de- 


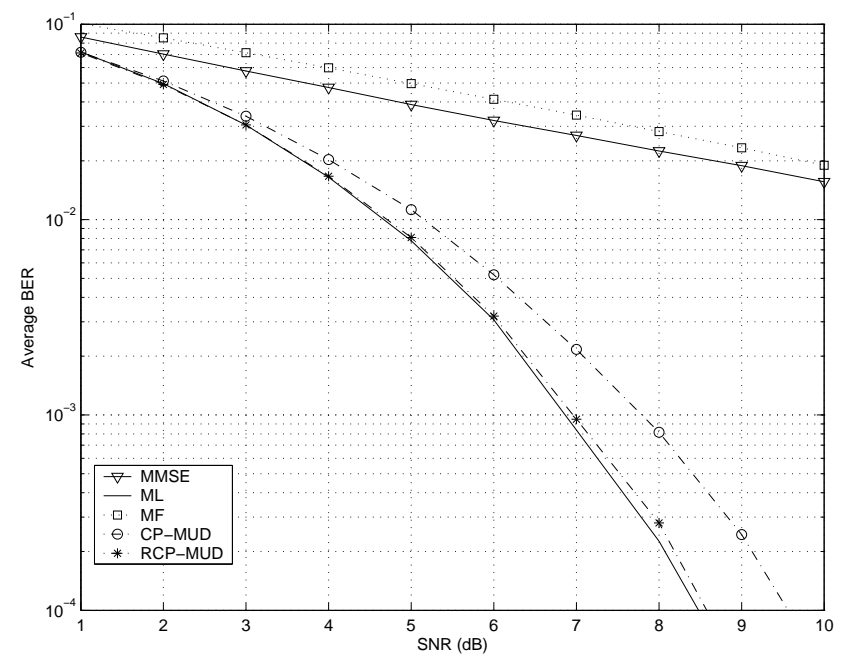

Fig. 1. BER performance of eight users in an AWGN channel.

tector with considerably reduced computational complexity.

\section{REFERENCES}

[1] I. Oppermann, M. Hämäläinen, and J. Iinatti, UWB Theory and Applications, John Wiley \& Sons, 2004.

[2] M. Z. Win and R. A. Scholtz, "Ultra-wide bandwidth time-hopping spread-spectrum impulse radio for wireless multiple-access communications," IEEE Trans. Commun., vol. 48, pp. 679-691, Apr. 2000.

[3] J. R. Foerster, "The performance of a direct-sequence spread ultrawideband system in the presence of multipath, narrowband interference and multiuser interference," Proc. IEEE Conf. UWB Sys. and Tech., May 2002, pp. 87-91.

[4] Q. Li and L. A. Rusch, "Multiuser detection for DSCDMA UWB in the home environment," IEEE J. Select. Areas in Commun., vol. 20, no. 9, pp. 1701-1711, Dec. 2002.

[5] R. J.-M. Cramer, M. Z. Win, and R. A. Scholtz, "Impulse radio multipath characteristics and diversity reception," IEEE Int. Conf. Commun., June 1998, pp. $1650-1654$

[6] S. Verdú, Multiuser Detection, Cambridge University Press, 1998.

[7] S. Boyd and L. Vandenberghe, Convex Optimization, Cambridge University Press, 2004.

[8] X. M. Wang, W.-S. Lu, and A. Antoniou, "Multiuser detectors for synchronous DS-CDMA systems based

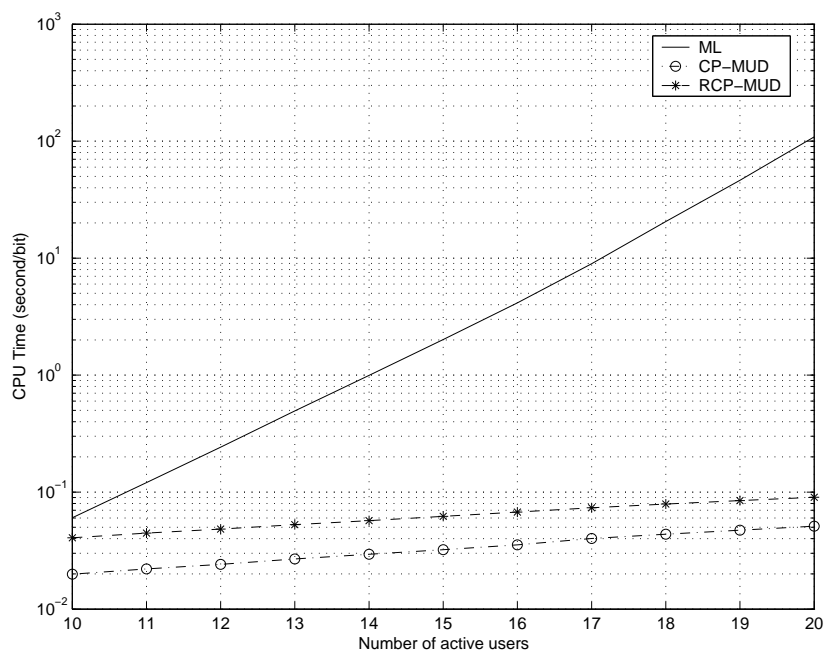

Fig. 2. Computational complexity of multiuser detectors.

on a recursive p-norm convex relaxation approach," accepted for publication in IEEE Trans. Circuits and Syst. I, Nov. 2004. 